\title{
Caracterización de las inteligencias múltiples en una muestra de estudiantes universitarios 1
}

\section{Multiple intelligences representation in a university students sample}

\author{
Humberto Closa回 \\ hclosas@hotmail.com \\ Idalia de Castrdc \\ idecastro@eco.unne.edu.ar
}

\author{
Laura Estigarribab \\ mlestigarribia@eco.unne.edu.ar \\ Gricela Rohded \\ grohde@eco.unne.edu.ar
}

Alicia Dusicke e

mad2607@yahoo.com

\begin{abstract}
Resumen
El rendimiento académico, un fenómeno multicausal, constituye la manifestación formal y expresa del grado de aprendizaje del alumno. Este trabajo toma como objeto de estudio la inteligencia desde la visión de Howard Gardner, recuperando la riqueza de su enfoque multidimensional y sus posibles proyecciones en el proceso de enseñanza-aprendizaje; tiene por objetivo realizar un análisis exploratorio de su representación en una comunidad de estudiantes universitarios de primer año, con el fin de describir las principales características aptitudinales de los jóvenes. La investigación se divide en dos partes: la primera corresponde al análisis teórico, mientras que la segunda se relaciona con el estudio empírico. La muestra, formada por alumnos de la asignatura Instituciones del Derecho Privado I, de la Facultad de Ciencias Económicas (FCE) de la Universidad Nacional del Nordeste (UNNE), fue elegida de manera no probabilística y por conveniencia; estuvo compuesta por 156 sujetos. Se trata de una investigación de estilo descriptivo, mediante encuesta. Se utilizó un test ad hoc de inteligencias múltiples, de 35 preguntas, organizadas en cinco ítems para cada una de las áreas de la inteligencia consideradas (Lingüística, Musical, Lógico-matemática, Espacial, Cinestésico-corporal, Intrapersonal e Interpersonal). En la fase empírica de este estudio, los análisis estadísticos descriptivos
\end{abstract}

\footnotetext{
${ }^{1}$ DOI: http://dx.doi.org/10.15332/s2027-3355.2017.0002.08

Closas, H., Estigarriba, L., De castro, i., Rohde, G. y Dusicka,A. (2017) Caracterización de las inteligencias múltiples en una muestra de estudiantes universitarios. Comunicaciones en Estadística, 10(2), 345-358.

a Profesor, Universidad Nacional del Nordeste, Argentina

${ }^{\mathrm{b}}$ Profesora, Universidad Nacional del Nordeste, Argentina

${ }^{c}$ Profesora, Universidad Nacional del Nordeste, Argentina

dProfesora, Universidad Nacional del Nordeste, Argentina

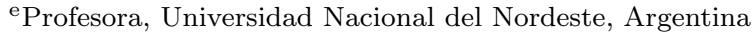


de fiabilidad y factorial permitieron reconocer que el cuestionario utilizado puede considerarse un instrumento confiable y que posee validez de constructo. El modelo que pudo ser identificado se encuentra conformado por tres factores a los que se ha denominado artístico, social y cuantitativo, en virtud de los tipos de inteligencias que integran a cada uno de ellos.

Palabras clave: Análisis psicométricos, dimensiones de la inteligencia, estudiantes universitarios.

\begin{abstract}
Academic performance is a multicausal phenomenon which clearly shows the learning levels of students. The current research aims to study intelligence as seen by Howard Gardner's theory, the importance of its multidimensional approach, and its possible applications on the teaching-learning process. It aims to make an exploratory analysis of its representation to describe the main aptitudinal characteristics of freshmen college students. The research is divided into two parts. The first part includes a theoretical analysis, and the second part includes the corresponding empirical study. The study group consists of 156 university students taking a course on Private Law I at the School of Economic Sciences (FCE) of Universidad Nacional del Nordeste (UNNE). This is a descriptive survey design study in which an ad hoc multiple intelligence test was used. It consists of 35 questions organized in 5 items for each considered area (Linguistic, Musical, Logical-Mathematical, Spatial, Bodily-kinesthetic, Intrapersonal and Interpersonal). The empirical stage of this study, the descriptive statistical analysis of reliability and the factorial validity measurement showed that the questionnaire used proved to be a reliable instrument, and that it had construct validity. This tri-factorial model consists of artistic, social and quantitative factors regarding the types of intelligences involved.
\end{abstract}

Keywords: Intelligence dimensions, psychometric analysis, university students.

\title{
1. Introducción
}

El proceso de interacción con el alumno, incluye dos etapas que, si bien están lógicamente vinculadas, son independientes entre sí; el aprendizaje (en sentido amplio), y su demostración objetiva (aprobación de evaluaciones). El rendimiento académico constituye la manifestación formal y expresa del grado de aprendizaje del alumno y se infiere tradicionalmente de las calificaciones. Representa un fenómeno multicausal, que puede ser abordado tomando en cuenta distintas variables, sus relaciones y las consecuencias que en él puedan tener. Este trabajo toma como objeto de estudio el constructo inteligencia, desde el desarrollo teórico de Howard Gardner.

Etimológicamente, el término viene del latín intelligentia y, en sentido amplio, sus distintas acepciones refieren a la comprensión; tienen que ver con capacidades para aprender o adaptarse de manera exitosa, como también con cuestiones 
de conocimiento adquirido. Howard Gardner, define la inteligencia como "la capacidad de resolver problemas, o de crear productos, que sean valiosos en uno o más ambientes culturales" (Gardner, 1993, p. 5). Postula que los seres humanos tienen una serie de habilidades relativamente independientes y, por ello, perciben el mundo en formas diferentes e igualmente importantes que configuran un perfil cognitivo único. A partir de su obra: Estructuras de la mente, se reseñan brevemente cada una de las siete dimensiones iniciales detectadas por Gardner (1993): Lingüística: Involucra la posibilidad de pensar en palabras y utilizar el lenguaje para comprender y expresar significados complejos. Musical: Se trata de la destreza para cantar, ejecutar un instrumento, leer, escribir o comentar patrones musicales. Lógico-matemática: es la competencia para manejar problemas a partir del dominio de un pensamiento abstracto y general. Espacial: es la capacidad de percibir con exactitud el mundo visual, para realizar transformaciones y modificaciones a las percepciones iniciales propias. Cinestésico-corporal: importa la pericia para utilizar el propio cuerpo, controlando sus movimientos, lo que requiere la coordinación de una variedad de componentes neurales y musculares. Personal: compuesta por dos formas de manifestación, intrapersonal e inter-personal. La primera, constituye la aptitud de autoconocimiento y reflexión acerca de las propias emociones y sentimientos. La segunda, es la cualidad que permite notar y establecer distinciones entre otros individuos y, en particular, entender sus estados de ánimo, temperamentos, motivaciones e intenciones. Estos dos aspectos (i.e., intra e interpersonal) están interrelacionados, puesto que guarda íntima correspondencia el auto comprensión con la posibilidad de percepción de los demás individuos. Son facultades de procesamiento de información, hacia adentro y hacia afuera. Después de haber revisado las siete inteligencias, es posible sostener, siguiendo a Gardner (1993), que pueden agruparse, en las siguientes dimensiones: a) relacionadas con objetos: espacial, lógico-matemática y cinestésico-corporal; están sujetas a un control físico ejercido sobre la estructura y las funciones de los objetos particulares en contacto los individuos; b) libres de objetos: lingüística y musical, que pueden desarrollarse mejor, en relación con el sistema auditivo oral y visual, aunque no dependen exclusivamente de ellos; c) personales: el conocimiento de sí mismo, de otras personas y de representaciones e interpretaciones culturales. Larivée (2010), recuperó numerosas críticas que se han realizado a esta teoría, agrupándolas en nueve categorías:

- uso del término "inteligencia"

- el carácter científico

- la originalidad del hallazgo

- los criterios para discriminar cada clase (de inteligencia)

- el funcionamiento independiente de cada tipo

- la importancia relativa que tienen

- su medición utilizando una evaluación contextualizada que reemplaza las pruebas estandarizadas de coeficiente intelectual 
- su vinculación con el constructo "factor g" (o factor general de inteligencia) que controla las habilidades cognitivas

- su aplicación por grupos.

Sin embargo, la mayor crítica efectuada hacia la teoría de Gardner es la insuficiencia de evidencia rigurosa, ante los escasos análisis empíricos propios sobre los que se apoya. Por ello, sus detractores consideran que sólo ofrece una opinión anecdótica en sus explicaciones. Pérez (2013) han sostenido que la teoría no posee instrumentos específicamente desarrollados para efectuar mediciones de manera objetiva y estandarizada. Asimismo expresan, "se han diseñado autoinformes que evalúan habilidades, preferencias y autoeficacia relacionadas con los constructos de la teoría, tales como la Escala de Evaluación del Desarrollo de las Inteligencias Múltiples MIDAS (Shearer 1999); el Perfil de Inteligencias Múltiples del Estudiante SMIP (Chan 2001) y el Inventario de Autoeficacia para Inteligencias Múltiples IAMI (Pérez 2001). Si bien estos instrumentos pueden facilitar la elaboración de perfiles relacionados con inteligencias múltiples y son útiles en algunos contextos aplicados (i.e., orientación vocacional, identificación de talento), adolecen de las limitaciones de este tipo de pruebas: a) no pueden ser aplicados en niños pequeños, y b) los individuos pueden sobreestimar o subestimar sus habilidade" (Pérez 2013). A pesar de las críticas mencionadas y en función de lo expuesto, se aborda este análisis desde las distintas dimensiones de la inteligencia provistas por la teoría inicial de Gardner. Se propone realizar un análisis exploratorio de su representación en una comunidad de estudiantes universitarios de primer año, a cuyo efecto se ha evaluado la fiabilidad y validez del instrumento aplicado, para conseguir la descripción de las principales características aptitudinales de los participantes.

El instrumento fue empleado previamente en numerosos contextos, y con distintos propósitos tales como el diagnóstico psicopedagógico, identificar preventivamente posibles dificultades de aprendizaje y al mismo tiempo potenciar aquella inteligencia dominante (Bandera 2012). También, como instrumento de auto conocimiento del nivel de desarrollo de cada inteligencia y qué actividades pueden ser fortalecidas, en beneficio del aprendizaje (Coutiño 2015). Asimismo, fue utilizado como test de aplicación de la teoría de las inteligencias múltiples en las prácticas docentes (Navarrete 2011); y, extendido a 40 preguntas, contemplando la octava categoría Naturalista, para establecer una relación entre: diversos tipos de inteligencia y rendimiento académico de estudiantes de $5^{\circ}$ a $7^{\circ}$ grado (Carreño 2013), o para evaluar inicialmente las competencias y características educativas del alumnado (García 2010).

Este estudio, descriptivo y psicométrico, constituye un paso necesario para describir, en un nuevo trabajo que será desarrollado posteriormente, las relaciones estadísticamente significativas entre los tipos de inteligencia y el rendimiento académico de los alumnos, en distintas áreas de conocimiento. 


\section{Diseño de la investigación}

Es una investigación, no experimental y exploratoria. Se obtuvieron datos primarios acerca de las preferencias de los estudiantes, mediante una encuesta que las asocia con las dimensiones de la inteligencia. Es una investigación de línea cuantitativa y de corte transversal. Los siete tipos de inteligencia, o variables latentes, que serán analizadas cuantitativamente en este estudio, corresponden a la formulación inicial de la teoría de Gardner: Lingüística, Musical, Lógico-matemática, Espacial, Cinestésico-corporal, Intrapersonal e Interpersonal. En este trabajo no será considerada la dimensión Naturalista, por estimar que la misma no resulta relevante a los fines del presente análisis.

\section{Materiales y método}

\subsection{Participantes}

Debido a que el interés radica en trabajar con la totalidad de los estudiantes de la asignatura: Instituciones del Derecho Privado I de la Facultad de Ciencias Económicas (FCE) de la Universidad Nacional del Nordeste (UNNE); la muestra fue seleccionada por conveniencia, y es claramente no probabilística. La materia mencionada se encuentra en el primer año del currículo y se dicta en el primer cuatrimestre de las carreras de Contador Público, Licenciatura en Economía y Licenciatura en Administración, de la FCE-UNNE. Concretamente, la muestra estuvo compuesta por 4 grupos-clase, los que representaban un total de 156 sujetos, 93 mujeres (59.62\%) y 63 hombres (40.38\%), con una media de 19.70 años y desviación estándar de 3.22 .

\subsection{Procedimiento}

La recolección de datos se realizó en una única sesión. Se les informó a los alumnos participantes que la aplicación del instrumento en cuestión respondía a un proyecto de investigación relacionado con temas de rendimiento académico, que tiene la intención de aportar propuestas de intervención. También se les indicó la importancia de responder sinceramente a los distintos temas planteados. Asimismo se les informó que sus respuestas tendrían un carácter estrictamente confidencial y que la participación en el estudio era voluntaria. La aplicación del cuestionario la efectuaron los propios docentes de la asignatura acompañados por algunos autores del presente estudio, al inicio de cada clase y con el margen de tiempo adecuado en virtud de las consultas formuladas en la prueba (en promedio 20 minutos). En conjunto se obtuvieron datos de 156 estudiantes. Posteriormente, se digitalizaron

y organizaron los datos recolectados en un formato electrónico, su procesamiento se realizó utilizando el software IBM SPSS Statistics 22. 


\subsection{Materiales}

Para cumplir con el objetivo propuesto y recoger los datos relativos al tema bajo estudio se utilizó un test de inteligencias múltiples. Se realizó una adaptación de los enunciados al contexto. El cuestionario consta de 35 afirmaciones, organizadas en 7 dimensiones de 5 ítems cada una: Lingüística (e.g., Me resulta fácil decir lo que pienso en el curso de una discusión o debate), Musical (e.g., Sé interpretar, o antes sabía, un instrumento musical), Lógico-matemática (e.g., Puedo sumar o multiplicar mentalmente con rapidez), Espacial (e.g. Prefiero hacer un dibujo que explicarle a alguien como tiene que llegar a un lugar), Cinestésico-corporal (e.g., Aprendo rápido a bailar un ritmo nuevo), Intrapersonal (e.g., Si estoy enojado/a o contento/a generalmente sé exactamente por qué) e Interpersonal (e.g., Me gusta reunir grupos de personas en una fiesta o en un evento). Para responder a cada una de las afirmaciones los estudiantes debían escribir una $\mathrm{V}$ (verdadero) o una $\mathrm{F}$ (falso), según estaban de acuerdo o desacuerdo con el respectivo enunciado.

\subsection{Análisis de datos}

La evaluación cualitativa del instrumento utilizado fue realizada por profesores de los Departamentos de Derecho, Matemática, Contabilidad y Administración de la FCE de la UNNE, en cuanto a los siguientes aspectos: a) pertinencia del contenido de los ítems propuestos (indicadores subjetivos de validez), y b) conformación del cuestionario en su conjunto (indicadores de la validez factorial o estructural). Las apreciaciones formuladas por un grupo de cinco docentes acerca del test objeto de evaluación tuvieron una amplia coincidencia. Sin duda, los análisis realizados en la línea de validez cualitativa (i.e., juicio de expertos y grado de acuerdo) fueron sumamente valiosos, a fin de minimizar los márgenes de error del instrumento de medición al momento de su utilización en nuestro contexto. De hecho, la validez de una prueba es un indicador del grado en que ésta es capaz de medir lo que realmente pretende medir (Hernández 2010), por lo que resulta necesaria y relevante su evaluación cualitativa inicial, así como su evaluación cuantitativa en la etapa empírica de la investigación (análisis psicométricos).

Con la base de datos se realizaron diferentes análisis estadísticos pertenecientes al dominio de la psicometría (correlación dimensión-total corregida y consistencia interna), también de la estadística descriptiva e inferencial (algunos estadísticos centrales y de dispersión, análisis correlacionales bivariados, análisis factorial; para las pruebas de hipótesis, utilizamos la medida p-valor), tienen el propósito final de aportar resultados que ayuden a lograr el objetivo de esta investigación. En efecto, los diversos cálculos realizados permitieron por un lado, conocer el comportamiento de cada una de las dimensiones que componen la prueba y, por otro, la fiabilidad y la validez de constructo del instrumento. 


\section{Resultados}

En atención al propósito de esta investigación y a los análisis estadísticos mencionados en el apartado anterior, se presentan de forma sintética los resultados de aquellos indicadores que nos han parecido más convenientes para caracterizar la muestra en el total de la escala y en las siete dimensiones que conforman la prueba utilizada.

\subsection{Estudios iniciales de los ítems del cuestionario aplicado}

En la tabla 1, pueden apreciarse los valores hallados para cada una de las dimensiones, así como para el conjunto de las mismas, en cuanto a puntuaciones directas, media y desviación estándar, los que resultaron razonables y se encuentran en el rango de medidas que se esperaba obtener, teniendo en cuenta el contexto en el que se desarrolla este estudio. El valor medio más alto se obtuvo en la inteligencia Intrapersonal $M=3.97, D E=1.03)$, en tanto que el menor valor medio resultó en la inteligencia Espacial $(M=2.09, D E=1.16)$. La utilidad de estos estadísticos reside en que nos ayudan a comprender de qué manera se encuentran distribuidos los datos de la muestra, en relación con algunas características aptitudinales de los sujetos participantes.

Por otra parte, las puntuaciones totales en cada una de las áreas muestran correlaciones corregidas aceptables con las puntuaciones totales en la prueba (sumatoria de los ítems que componen las dimensiones, excluidos aquellos que integran la dimensión cuya asociación se evalúa), puesto que en todos los casos superan el valor de referencia 0.20 (Kline 2000); observándose la más alta (0.46) en el área denominada Intrapersonal, mientras que la más baja (0.36) resultó para la dimensión Lógico-matemática. Este coeficiente, denominado índice de homogeneidad corregido, puede considerarse un indicador del grado de discriminación que posee la dimensión; cuanto más alta y positiva sea la correlación, mayor será la capacidad de la dimensión para discriminar los sujetos respecto del constructo o concepto que se pretende medir con la escala objeto de interés.

Respecto de los indicadores $\alpha$ de Cronbach, cuando se excluye la dimensión que se desea medir, podemos señalar que el menor valor hallado (0.56) corresponde al área Musical, en tanto que el mayor coeficiente obtenido (0.62) correspondió a la dimensión Lógico-matemática. Cabe mencionar que cuanto más bajo resulte este índice, más se pone en evidencia el aporte de la dimensión a la consistencia interna de la escala (se recuerda que el coeficiente se calcula a partir de los ítems que conforman las restantes dimensiones; es decir, sin la participación de aquellos que pertenecen a la dimensión cuya contribución a la fiabilidad de la prueba se desea medir). La fiabilidad, es una característica fundamental en cualquier test, y una de las formas de evaluarla es precisamente a través del coeficiente $\alpha$ de Cronbach, el cual indica la precisión o estabilidad de los resultados; señala la cuantía en 
que las medidas de la prueba están libres de errores casuales o aleatorios. En

Tabla 1: Estadísticos descriptivos y de fiabilidad de las dimensiones de la prueba. Fuente: elaboración propia.

\begin{tabular}{|l|l|l|l|l|l|l|}
\hline Dimensión & $\begin{array}{l}\text { Num. } \\
\text { de } \\
\text { ítems }\end{array}$ & $\begin{array}{l}\text { Puntuaciones } \\
\text { directas }\end{array}$ & Media & DE & $\begin{array}{l}\text { Correlación } \\
\text { dimen- } \\
\text { sión-total } \\
\text { corregida }\end{array}$ & $\begin{array}{l}\alpha \text { de Cron- } \\
\text { bachsin } \\
\text { dimensión } \\
\text { la }\end{array}$ \\
\hline Lingüística & 5 & $\begin{array}{l}\text { Mín. }=1 \text { Máx. } \\
=5\end{array}$ & 3.02 & 1.13 & 0.44 & 0.58 \\
\hline Mún. $=0$ Máx. & 3.20 & 1.38 & 0.44 & 0.56 \\
\hline Lóg.matem. & 5 & $\begin{array}{l}\text { Mín. }=1 \text { Máx. } \\
=5\end{array}$ & 3.53 & 1.25 & 0.36 & 0.62 \\
\hline Espacín. $=0$ Máx. \\
$=5$
\end{tabular}

atención a las consideraciones realizadas en los dos párrafos anteriores, acerca de los coeficientes correlación dimensión-total corregida y $\alpha$ de Cronbach excluida la dimensión que se valora, es posible señalar a partir de los datos analizados que las áreas Intrapersonal y Musical son las que poseen mayor relevancia en el marco general del análisis de fiabilidad de la prueba objeto de evaluación. No obstante, los coeficientes relativos a las demás dimensiones que integran el instrumento están próximos a los obtenidos para las sub escalas mencionadas, por lo que puede sostenerse que todas las áreas resultan relevantes para medir el constructo de interés. En efecto, la consistencia interna calculada para el conjunto de las siete dimensiones puede considerarse suficiente, para un contexto de tipo exploratorio en las primeras fases de una investigación, como es nuestro caso, puesto que el coeficiente alfa encontrado $(\alpha=0.62)$ verifica el criterio de algunos autores de estar entre 0.50 y 0.60 , o hallarse por encima de este último valor (Huh 2006). [

\subsection{Análisis correlacionales bivariados}

En este apartado llevaremos a cabo análisis correlacionales entre las siete dimensiones que integran la prueba. La primera razón por la que se realizan estos estudios radica en el hecho de que los coeficientes que se obtengan permitirán en principio confirmar, o no, las relaciones lineales que se presume existen entre los distintos

\footnotetext{
${ }^{1}$ Calcular el coeficiente de fiabilidad en cada nueva muestra, y no apoyarse en la obtenida en otros estudios como aval de la fiabilidad del instrumento, es una de las recomendaciones de la American PsychologicalAssociation (Wilkinson 1999, Association 2001); también sugerido por la política editorial de importantes revistas (Thompson 1994).
}

Comunicaciones en Estadística, diciembre 2017, Vol. 10, No. 2 
tipos de inteligencia que conforman el cuestionarioobjeto de interés. El segundo motivo de los análisis correlacionales reside en que está proyectado a continuación realizar un análisis factorial exploratorio con dichas categorías (a efectos de evaluar la validez de constructo de la prueba) y es siempre de utilidad examinar previamente las relaciones lineales que las mismas presentan (en principio, con el fin de seleccionar adecuadamente el procedimiento de rotación que se empleará al momento de generar la matriz de pesos factoriales). Así pues, de acuerdo con los valores de la tabla 2 , puede afirmarse que algunas de las dimensiones que componen el cuestionario tienen correlaciones positivas y estadísticamente significativas $(r=0.16$ a $0.18, p<0.05 ; r=0.23$ a $0.25, p<0.01 ; N=156)$. En este contexto, el coeficiente de correlación más bajo se presenta entre las inteligencias Lingüística e Interpersonal $(r=0.16, p<0.05)$, mientras que el más alto se observa entre las dimensiones Lógico-matemática y Espacial $(r=0.25, p<0.01)$. Más allá del

Tabla 2: Matriz de correlaciones. Fuente: elaboración propia.

\begin{tabular}{|c|c|c|c|c|c|c|c|}
\hline Inteligencia & $\begin{array}{l}\text { Lingüísti- } \\
\text { ca }\end{array}$ & Musical & $\begin{array}{l}\text { Lógico } \\
-\quad \text { ma- } \\
\text { temáti- } \\
\text { ca }\end{array}$ & Espacial & $\begin{array}{l}\text { Cinest.- } \\
\text { corpo- } \\
\text { ral }\end{array}$ & $\begin{array}{l}\text { Intra- } \\
\text { perso- } \\
\text { nal }\end{array}$ & $\begin{array}{l}\text { Inter } \\
\text { perso- } \\
\text { nal }\end{array}$ \\
\hline Lingüística & 1 & 0.08 & 0.01 & $0.21^{* *}$ & -0.10 & $0.17^{*}$ & $0.16^{*}$ \\
\hline Musical & & 1 & 0.05 & -0.13 & $0.23^{* *}$ & 0.11 & 0.10 \\
\hline Lóg.--matem. & & & 1 & $0.25^{\text {** }}$ & 0.07 & -0.04 & -0.03 \\
\hline Espacial & & & & 1 & 0.01 & 0.06 & -0.08 \\
\hline Cin.--corporal & & & & & 1 & 0.09 & $0.17^{*}$ \\
\hline Intrapersonal & & & & & & 1 & $0.18^{*}$ \\
\hline Interpersonal & & & & & & & 1 \\
\hline
\end{tabular}

valor que poseen los índices hallados, lo relevante es que algunos resultaron significativos, estadísticamente hablando; es decir, pudo ser contrastada - a partir de los datos de la muestra - la existencia de correlaciones lineales bivariadas entre las distintas inteligencias que componen el cuestionario aplicado. La observación de este hecho es sin duda importante puesto que permite reconocer grupos de dimensiones (pares y ternas) que, en cierta forma, nos ayudan a perfilar la estructura factorial que subyace al instrumento objeto de interés, la cual se presenta a continuación.

\subsection{Análisis factorial}

Si bien, en una aproximación teórica y empírica puede decirse que las dimensiones medidas se encuentran agrupadas en determinados factores, emplearemos la técnica del análisis factorial a efectos de identificar claramente estas variables latentes; es decir, contrastar la validez de constructo o estructural del cuestionario aplicado. Siguiendo a (Comrey 1973), antes de realizar el análisis factorial exploratorio, se ha comprobado la adecuación de los datos para este tipo de estudio. Una síntesis de los resultados es la siguiente: a) determinante de la matriz de correlaciones (0.69) relativamente bajo; b) medida de adecuación de la muestra de Kaiser, Meyer y Olkin, índice $\mathrm{KMO}=0.71$, ubicado en un rango aceptable de potencial explicativo de las variables (Kaiser 1974); c) contraste de esfericidad de Bartlett con 2(21) 
$-55.83, p=0.00$. En vista de los valores obtenidos, en principio, el análisis factorial es una opción pertinente que nos ayudará a identificar los factores subyacentes al grupo de siete dimensiones que se analiza.

Para determinar la solución factorial hemos empleado el criterio clásico; esto es, la extracción de tantos factores como haya en la solución inicial con autovalores superiores a la unidad, lo que se realizó por el método de componentes principales y rotación varimax, en atención a los resultados obtenidos en los análisis correlacionales. Este procedimiento ha delimitado una estructura trifactorial que explica un $57.34 \%$ de la varianza total, lo que puede interpretarse como un porcentaje aceptable, puesto que para investigaciones que se realizan en áreas de psicología social lo recomendable es que la varianza acumulada se encuentre, al menos, entre el $50 \%$ y $60 \%$ (Henson 2006). Según los resultados del análisis efectuado el factor que permite explicar la mayor cantidad del total de la varianza de la muestra está integrado por las inteligencias Cinestésico-corporal y Musical (20.30\%), en este sentido el segundo factor se conforma por las áreas Lingüística, Intrapersonal e Interpersonal (19.06\%), mientras que en tercer lugar se encuentra el factor constituido por las dimensiones Lógico-matemática y Espacial (17.98\%). En la tabla 3 se recogen, para cada uno de los factores subyacentes, el valor propio, el porcentaje de varianza tanto explicada como acumulada, las inteligencias que los integran, sus comunalidades (proporción de varianza de la variable que puede ser explicada por el modelo factorial obtenido) y finalmente sus cargas factoriales o saturaciones (correlación entre la variable y el factor). Evidentemente, en atención a lo que

Tabla 3: Análisis factorial por componentes principales y rotación varimax. Fuente. elaboración propia.

\begin{tabular}{|c|c|c|c|c|c|c|}
\hline Factor & $\begin{array}{l}\text { Valor } \\
\text { propio }\end{array}$ & $\begin{array}{l}\text { Varianza } \\
\text { explica- } \\
\text { da }\end{array}$ & $\begin{array}{l}\text { Varianza } \\
\text { acumu- } \\
\text { lada }\end{array}$ & Inteligencia & Comunalide & dSaturación \\
\hline 1. Artístico & 1.51 & 20.30 & 20.30 & $\begin{array}{l}\text { Cin.--corporal } \\
\text { Musical }\end{array}$ & $\begin{array}{l}0.59 \\
0.48\end{array}$ & $\begin{array}{l}0.74 \\
0.68\end{array}$ \\
\hline 2. Social & 1.36 & 19.06 & 39.36 & $\begin{array}{l}\text { Lingüística } \\
\text { Intrapersonal } \\
\text { Interpersonal }\end{array}$ & $\begin{array}{l}0.65 \\
0.48 \\
0.45\end{array}$ & $\begin{array}{l}0.77 \\
0.64 \\
0.49\end{array}$ \\
\hline 3. Cuantitativo & 1.15 & 17.98 & 57.34 & $\begin{array}{l}\text { Lóg. matemática } \\
\text { Espacial }\end{array}$ & $\begin{array}{l}0.69 \\
0.68\end{array}$ & $\begin{array}{l}0.78 \\
0.73\end{array}$ \\
\hline
\end{tabular}

antecede, podemos señalar que el modelo factorial ha verificado un aspecto que es estratégico en toda acción de modelado estadístico: la "parsimonia", explicación más simple entre varias posibles. En efecto, la estructura obtenida, por un lado, posee un reducido número de factores $\mathrm{y}$, por otro, todos ellos son susceptibles de interpretación sustantiva. Así pues, el Factor 1, denominado Artístico representaría a los estudiantes cuyo perfil intelectual y cognitivo se encuentra vinculado con la posibilidad de cantar con mayor precisión y expresividad, leer, escribir o comentar patrones musicales. También con habilidades y capacidades para la utilización competente del cuerpo. En cuanto al Factor 2, llamado Social, describiría a 
los alumnos cuyas capacidades se hallan relacionadas con la posibilidad de pensar en palabras y utilizar el lenguaje para la comprensión y expresión de significados complejos; además la aptitud del autoconocimiento reflexivo de sus propias emociones y sentimientos como así también la habilidad de entender los estados de ánimo, temperamentos, motivaciones e intenciones de otros individuos. Por último, el tercer Factor, designado Cuantitativo representaría el perfil cognitivo de los estudiantes que se encuentra relacionado con una forma de pensamiento que abarca lo abstracto y general, demostrando habilidades para manejar determinadas clases de problemas lógico matemáticos y percibir con exactitud el mundo visual, con habilidades para realizar transformaciones y modificaciones a las percepciones iniciales propias. Sería conveniente señalar que nos hemos encontrado referencias bibliográficas sobre estudios que hayan obtenido, a partir del cuestionario aplicado, una estructura factorial similar, tampoco diferente, a la lograda en este trabajo. Sin embargo, en virtud de los distintos indicadores hallados a lo largo de esta investigación, podemos sostener que la escala bajo estudio es fiable y presenta validez de constructo; esto es, sería de utilidad para medir razonablemente las inteligencias múltiples.

\section{Discusión y conclusiones}

En el presente estudio nos propusimos realizar un análisis exploratorio, en una comunidad de estudiantes universitarios de primer año, de la caracterización de las distintas dimensiones del género inteligencia provista por la teoría inicial de Gardner; lo que ha posibilitado evaluar la fiabilidad y validez del instrumento aplicado, así como describir las principales características aptitudinales de los jóvenes participantes. En efecto, la investigación realizada permitió, en primer lugar, analizar el comportamiento de cada una de las variables latentes que integran el cuestionario empleado, las cuales representan las siete áreas de la inteligencia consideradas; lo que fue posible a partir de la aplicación de técnicas estadísticas descriptivas e inferenciales. En segundo término, se conocieron las características psicométricas del instrumento aplicado, lo que se logró en razón de los estudios de fiabilidad y validez, efectuados mediante análisis de correlación dimensión-total corregida y consistencia interna, como también a través de la técnica análisis factorial (método de componentes principales y rotación varimax). Lo que antecede ha sido el punto inicial que ha conducido a identificar y delinear distintos aspectos relativos a las aptitudes, capacidades y posibles competencias de los estudiantes que conforman la muestra y, por ende, conocer el perfil intelectual de los mismos. En razón de todo lo expuesto, el cuestionario utilizado es un instrumento confiable y válido que en nuestro contexto socio-cultural y académico, ha permitido caracterizar las distintas dimensiones de la inteligencia por las que hemos optado y agruparlas en una estructura trifactorial integrada por los constructos que subjetivamente hemos denominado Artístico, Social y Cuantitativo.

Resulta interesante resaltar que los factores Social y Cuantitativo son los que po- 
seen las inteligencias con mayor comunalidad y saturación, Lingüística (0.65 y0.77) y Lógico-matemática (0.69 y 0.78), respectivamente (véase tabla 3), lo que era de esperar en virtud del perfil académico y profesional de las carreras que se imparten en el ámbito de la FCE-UNNE. Si bien los resultados logrados indican ciertas evidencias acerca de las bondades de este estudio, es necesario señalar algunas limitaciones que hemos observado. En primer lugar, los sujetos de la presente investigación fueron alumnos pertenecientes a un centro académico específico, lo que no permitiría hacer inferencias amplias de los resultados alcanzados sobre otros estudiantes universitarios. A su vez, los participantes no fueron seleccionados en forma aleatoria, por lo que en general sería poco recomendable extender las apreciaciones que se formulen sobre otras poblaciones no representadas en la muestra.

El trabajo llevado a cabo evidenció el interés del desarrollo de futuras investigaciones en torno a evaluar la validez predictiva del cuestionario aplicado respecto de los resultados educativos, para lo cual podría plantearse un estudio mediante una técnica estadística multivariante explicativa o de dependencia. En el modelo que se proponga, por cierto, el rendimiento académico (utilizando las calificaciones de los estudiantes para conformarlo), sería la variable dependiente, mientras que las distintas dimensiones de la inteligencia conformarían el grupo de variables independientes, explicativas o predictoras.

A pesar que la muestra utilizada en esta investigación es acotada y posee ciertas particulares, por lo que, según fue indicado, los resultados expuestos deberían considerarse con cierta cautela, pensamos que el estudio realizado debe ser reconocido como un paso adelante en el abordaje del tema objeto de interés y, consecuentemente, un aporte a la comunidad científica y profesional del área de conocimiento, con posibles proyecciones en ámbitos de planificación y gestión psicoeducativa. Como última reflexión se indica que el hecho de haber logrado un modelo empírico en un determinado contexto educativo (estudiantes de Ciencias Económicas en Universidad Nacional de gestión pública de la zona noreste de Argentina), da origen a contar con un nuevo marco de referencia, lo cual permite ampliar la metodología utilizada y el instrumento de medida aplicado. La inteligencia y sus múltiples dimensiones, representan un concepto relevante debido a su probable implicancia en los resultados académicos, por lo que deberían incrementarse sus líneas de investigación a efectos de lograr un mayor desarrollo sobre su conocimiento.

Recibido: 24 de Junio de 2016 Aceptado: 30 de Agosto de 2017

\section{Referencias}

Association, A. P. (2001), 'Publication manual of the american psychological association'. 
Bandera, B. (2012), 'Elaboración y aplicación de un plan de intervención psicopedagógica en dos niños de 9 y 11 años, de la escuela atenas del ecuador con problemas en el área académica y emocional'.

*http://dspace.uazuay.edu.ec/bitstream/datos/4443/1/09002.pdf

Carreño, V. (2013), 'El desarrollo de las inteligencias múltiples y su incidencia en el rendimiento académico de los niños del programa de desarrollo comunitario "caminemos juntos" del barrio víctor emilio valdivieso, de la ciudad de loja durante el periodo 2012-2013'.

*https://dspace.unl.edu.ec/jspui/handle/123456789/2951

Chan, D. (2001), 'Assessing giftedness of chinese secondary students in hong kong: A multiple intelligences perspective', High Ability Studies (2), 215-234.

Comrey, A. (1973), 'A first course en factor analysis', New York: Academic Press

Coutiño, A. (2015), 'Nuestras inteligencias múltiples. aprender a aprender. universidad veracruzana'.

*http://www.uv.mx/coatza/ cadi/files/2013/10/InteligenciasMultiples.pdf

García, I. (2010), 'Sistemas de evaluación', Universidad de Salamanca . *http://www.eumed.net/libros-gratis/2010b/687/

Gardner, H. (1993), 'Estructuras de la mente. la teoría de las inteligencias múltiples (2a. ed.)', Colombia: Fondo de Cultura Económica .

Henson, K. y Roberts, J. (2006), 'Use of exploratory factor analysis in published research: Common error and some comment on improved practice', Educational and Psychological Measurement (3), 393-416.

Hernández, R. y Baptista, P. (2010), 'Metodología de la investigación (5a. ed.)', México, D.F.: McGraw-Hill .

Huh, J. y Reid, L. (2006), 'Perceived third person effects and consumer attitudes in prevetting and banning dtc advertising', Journal of Consumer Affairs pp. 90116.

Kaiser, H. (1974), 'An index of factorial simplicity', Psychometrika pp. 31-36.

Kline, P. (2000), 'The handbook of psychological testing (2a.ed.)', London: Routledge.

Larivée, S. (2010), 'Las inteligencias múltiples de gardner. ¿descubrimiento del siglo o simple rectitud política?', Revista Mexicana de Investigación en Psicología (2), 115-126.

Navarrete, P. y Queutre, J. (2011), 'Teoría de las inteligencias múltiples en educación: una revisión crítica', Universidad Católica de Temuco .

*http://www.researchgate.net/publication/282493551 
358Humberto Closas, Laura Estigarriba, Idalia de Castro, Gricela Rohde \& Alicia Dusicka

Pérez, E. (2001), 'Construcción de un inventario de autoeficacia para inteligencias múltiples', Universidad Nacional de Córdoba, Argentina .

Pérez, E. y Medrano, L. (2013), 'Teorías contemporáneas de la inteligencia. una revisión crítica de la literatura', Psiencia (2), 105-118.

Shearer, B. (1999), 'The midas challenge!', USA: Greyden Press .

Thompson, B. (1994), 'Guidelines for authors', Educational and Psychological Measurement pp. $837-847$.

Wilkinson, L. y. T. F. o. S. I. A. B. o. S. A. (1999), 'Methods in psychology journals: Guidelines and explanations', American Psychologist (8), 594-604. 